\title{
Gadolinium-haltige Kontrastmittel in der Magnetresonanztomografie
}

\author{
Eine Patienteninformation der Deutschen Röntgengesellschaft e. V. (DRG) und des Berufsverban- \\ des der Deutschen Radiologen e. V. (BDR)
}

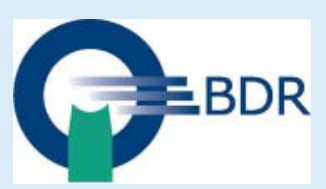

Die Magnetresonanztomografie (MRT) ist seit mehr als zwei Jahrzehnten eine sichere und unverzichtbare Bildgebungsmethode in der medizinischen Diagnostik. Sehr häufig erfolgt die Untersuchung ohne eine zusätzliche Kontrastmittelbeigabe. Für bestimmte Fragestellungen und zur weiteren Charakterisierung krankhafter Veränderungen ist jedoch oftmals die Gabe eines Kontrastmittels erforderlich. Dieses verbessert die Aussagekraft in erheblichem und nicht selten auch in entscheidendem Maße. Zum Einsatz kommen dabei Kontrastmittelpräparate, die Gadolinium enthalten und sich u. a. in ihrer chemischen und physikalischen Struktur unterscheiden, d.h. entweder linear (kettenartig) oder makrozyklisch (ringförmig) aufgebaut sind. Kontrastmittel werden überwiegend über die Nieren wieder ausgeschieden.

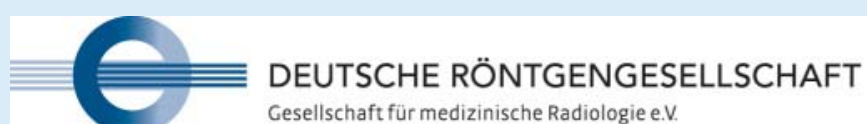

Gesellschaft für medizinische Radiologie e.V.

\section{Verträglichkeit}

Bei insgesamt sehr guter Verträglichkeit aller zugelassenen MRT-Kontrastmittel werden selbst allergische Reaktionen als häufigste Nebenwirkung nur sehr selten beobachtet. Die wichtigste Anwendungsbeschränkung der vergangenen Jahre bezog sich auf Patienten mit einer eingeschränkten Nierenfunktion, da in diesen Fällen die Ausscheidung der Kontrastmittel verzögert ist. Der Ausscheidungsprozess über die Niere kann insbesondere bei Patienten mit einer Niereninsuffizienz verlangsamt oder - bei wiederholter Anwendung eines MRKontrastmittels - auch unvollständig erfolgen, sodass Ablagerungen in Geweben möglich sind, die in sehr seltenen Fällen eine nephrogene systemische Fibrose (NSF) auslösen können. Bei geschätzt rund 400
Millionen Untersuchungen mit Kontrastmittel weltweit hat bei ca. 400 schwer nierenkranken Patienten eine wiederholte Kontrastmittelgabe zu einer NSF geführt. Nachdem Wissenschaftler, Aufsichtsbehörden und radiologische Gesellschaften umfassend reagiert haben, ist die Zahl der Neuerkrankungen deutlich zurückgegangen.

In jüngsten wissenschaftlichen Publikationen wurde über zerebrale Ablagerungen von Gadolinium in verschiedenen Regionen des Gehirns berichtet und zwar bei Patienten, die aufgrund von Tumorerkrankungen oder von entzündlichen Erkrankungen wie der Multiplen Sklerose wiederholt Kontrastmittel erhielten. Gemäß aktuellem wissenschaftlichen Kenntnisstand verursachen diese Ablagerungen keine Erkrankungen 
oder Symptome. Von vielen erfahrenen Forschergruppen weltweit werden derzeit weitere Untersuchungen durchgeführt.

\section{Sicherheit}

Eine Bewertung durch die Europäische Arzneimittelagentur (EMA) hat bestätigt, dass sich nach Anwendung von Gadolinium-haltigen Kontrastmitteln geringe Mengen Gadolinium im Gehirn ablagern. Dabei wurden bei der Gabe von linearen Gadolinium-haltigen Kontrastmitteln mehr Ablagerungen im Gehirn beobachtet als bei makrozyklischen Gadolinium-haltigen Kontrastmitteln. Hinweise für eine Schädigung der Patienten durch die Gadoliniumablagerung im Gehirn wurden nicht festgestellt. Da die langfristigen Risiken einer Gadoliniumablagerung im Gehirn unbekannt sind, empfahl die EMA das Ruhen der Zulassungen für intravenöse lineare Gadolinium-haltige Kontrastmittel in der EU mit Ausnahme der Wirkstoffe Gadoxetsäure und Gadobensäu- re, die weiterhin verfügbar bleiben, jedoch nur zur MRT-Bildgebung der Leber. Das Bundesinstitut für Arzneimittel und Medizinprodukte (BfArM) hat das Gutachten der EMA für rechtsverbindlich erklärt und ein Ruhen der Zulassungen für die betroffenen Kontrastmittel mit Stichtag 28.02.2018 angeordnet.

\section{Vertrauen}

Handlungsleitend für alle Radiologen in Deutschland ist das gemeinsame Ziel, ein Höchstmaß an diagnostischer Qualität mit der größtmöglichen Sicherheit für die Patienten zu verbinden. Bei MRT-Untersuchungen verbessern Kontrastmittel die Aussagekraft in erheblichem, nicht selten in entscheidendem Maße. In jedem Einzelfall gilt es daher, das Risiko, ohne MR-Kontrastmittel einen wichtigen, unter Umständen auch lebensbedrohlichen Befund zu übersehen, gegenüber den durch ihren Einsatz möglichen Nebenwirkungen abzuwägen.
Dabei befolgen die Radiologen zu Ihrer Sicherheit folgende Grundsätze:

- Der Einsatz eines Kontrastmittels erfolgt immer nach sorgfältiger Abwägung, ob sich dadurch zusätzliche wichtige Informationen gewinnen lassen.

- Es wird grundsätzlich die geringstmögliche Menge Gadolinium verabreicht.

- Eine Kontrastmittelgabe erfolgt immer unter Berücksichtigung Ihrer individuell bestehenden Risikofaktoren wie z. B. Nierenerkrankungen, Diabetes mellitus oder Allergien.

- Alle verwendeten Kontrastmittel sind nach internationalen und deutschen Richtlinien überprüft und als Arzneimittel zugelassen.

Ihre Radiologin, Ihr Radiologe stehen Ihnen selbstverständlich für weitere Fragen im persönlichen Aufklärungsgespräch gerne zur Verfügung. 